PharmacoEconomics \& Outcomes News 853, p15 - 16 May 2020

\title{
Demand for potentially hazardous COVID-19 treatments
}

Demand for unproven and potentially life-threatening treatments for COVID-19 infections appears to have been increased by public endorsements, according to findings of a study published in JAMA Internal Medicine.

Chloroquine and hydroxychloroquine were endorsed by Elon Musk on 16 March 2020 and by President Donald Trump on 19 March 2020. The demand for chloroquine and hydroxychloroquine for the prevention or treatment of COVID-19 in USA was assessed by investigating the proportion of Google searches each day between 1 February and 29 March 2020 which included the terms chloroquine or hydroxychloroquine in addition to the terms buy, order, Amazon, eBay or Walmart.

The query fraction of Google searches per 10 million for the purchase of chloroquine increased from 4.78 on 1 February (542 searches) to 26.90 on 16 March and 66.16 on 22 March (7506 searches), and then decreased to 19.19 on 29 March 29. Query fractions for the purchase of hydroxychloroquine increased from 4.35 on 1 February (494 searches) to 7.68 on 16 March, 79.37 on 22 March (9006 searches) and 31.95 on 29 March.

Overall, queries for chloroquine and hydroxychloroquine increased by $442 \%$ and $1389 \%$, respectively, after claims that they were effective for COVID-19 infections. The queries remained $212 \%$ and $1167 \%$ higher, respectively, after the first fatal poisoning was reported.

"Demand for chloroquine and hydroxychloroquine increased substantially following endorsements by high-profile figures and remained high even after a death attributable to chloroquine containing products was reported. In times of public health crises, therapies not supported by adequate evidence-such as would lead to US Food and Drug Administration approval—should not be touted by public figures," said the authors.

Liu M, et al. Internet Searches for Unproven COVID-19 Therapies in the United States. JAMA Internal Medicine : 29 Apr 2020. Available from: URL: http:// dx.doi.org/10.1001/jamainternmed.2020.1764 